\title{
Shape Synthesis in Mechanical Design
}

\author{
C. P. Teng, S. Bai, J. Angeles
}

The shaping of structural elements in the area of mechanical design is a recurrent problem. The mechanical designer, as a rule, chooses what is believed to be the "simplest" shapes, such as the geometric primitives: lines, circles and, occasionally, conics. The use of higher-order curves is usually not even considered, not to speak of other curves than polynomials. However, the simplest geometric shapes are not necessarily the most suitable when the designed element must withstand loads that can lead to failure-prone stress concentrations. Indeed, as mechanical designers have known for a while, stress concentrations occur, first and foremost, by virtue of either dramatic changes in curvature or extremely high values thereof. As an alternative, we propose here the use of smooth curves that can be simply generated using standard concepts such as non-parametric cubic splines. These curves can be readily used to produce either extruded surfaces or surfaces of revolution.

Keywords: Curve synthesis, cubic splines, optimum design, $G^{2}$-continuity.

\section{Introduction}

The problem of curve synthesis occurs frequently in mechanical design $[1,2,3,4,5,6]$. This problem arises whenever two flat - the case of a surface - or straight - the case of a line segments of a machine element are to be joined either to close an orifice smoothly or to join that segment to the machine frame. The reason why a smooth transfer is required lies in the need to prevent stress concentrations.

Mechanical designers over the years have designed machines with rectangular bores using circular arcs to round the corners. This is done with two purposes: (i) to avoid stress concentrations that would arise due to an inWnite curvature at the corner, and (ii) to ease the machining of the bore. The problem with circular arcs is that they provide only $G^{1}$-continuity. Second-order geometric continuity $G^{2}$, on the other hand, requires that the two curvatures coincide at the blending point; however, the curvature of the straight segment is zero, while that of the circular arc is the reciprocal of the radius of the circle, which is a finite quantity different from zero. The outcome is that stress concentrations are not eliminated because of the curvature discontinuity at the point of blending [7].

In this paper, a methodology is proposed aiming at the production of $G^{2}$-continuity at the blending of segments of two curves. The paper is based on the concept of curve synthesis, as first proposed in [1]. While the forgoing reference resorts to parametric cubic splines to synthesize geometric curves, we show in this paper that a geometric curve can also be synthesized using non-parametric splines, thereby streamlining the procedure.

\section{Problem statement}

First and foremost, a distinction is made between a curve representing the plot of a function and a geometric curve: while the slope of the former is bounded, the latter is not; moreover, a geometric curve can have cusps, can cross itself and need not be representable by analytic functions. The paper focuses on geometric curves, while resorting to non-parametric cubic splines.

Curve synthesis in this context is defined as: Given two curve segments $\Gamma_{a}$ and $\Gamma_{b}$ lying in the same plane, find a third curve segment $\Gamma_{c}$ that blends smoothly with both $\Gamma_{a}$ and $\Gamma_{b}$ at the blending points $A$ and $B$, with $G^{2}$-continuity.

Notice that we stated the search of a segment, as opposed to that of the segment, to emphasize that the problem admits multiple solutions. In fact, the problem admits infinitely many solutions. To pinpoint one particular solution, we must impose additional conditions. Many are possible, the one that we adopt here being that the segment sought $\Gamma_{c}$ be "as straight as possible." What this means is that we want the segment to have the smallest possible curvature. This requirement makes sense in design engineering, since a straight segment is the simplest shape to fabricate and, in the realm of structural engineering, the least likely to offer high bending moments if, for example, $\Gamma_{c}$ were to be the neutral axis of a beam. The challenge here is to formulate the synthesis problem in a standard form, e.g., one that would lead to an optimization problem.

To this end, we resort to a discretization of the curve. Many forms of curve discretization are available in the realm of geometric modeling, namely, Bézier curves, cubic splines, B-splines, non-uniform rational B-splines, or NURBS, and so on [8]. We limit the search to the simplest of these tools, namely, non-parametric cubic splines.

\section{Problem formulation using cubic splines}

In discretizing the problem at hand by means of cubic splines, we define $n+2$ supporting points $\left\{P_{k}\right\}_{0}^{n+1}$, the $k^{\text {th }}$ point having Cartesian coordinates $P_{k}\left(x_{k}, y_{k}\right)$. Shown in Fig. 1 is a sketch of the blending segments $\Gamma_{a}$ and $\Gamma_{b}$ by means of $\Gamma_{c}$, with $P$ denoting a generic point of the latter. Alternatively, points $\left\{P_{k}\right\}_{0}^{n+1}$ are defined by their polar coordinates $P_{k}\left(\rho_{k}, \theta_{k}\right)$. Henceforth, we define $P_{0}=A$ and $P_{n+1}=B$.

Now, let $A\left(\rho_{A}, \theta_{A}\right)$ and $B\left(\rho_{B}, \theta_{B}\right)$ be the polar coordinates of the blending points. Moreover, let

$$
\Delta \theta=\frac{\theta_{B}-\theta_{A}}{n+1}
$$

and define $\theta_{k}=\theta_{A}+k \Delta \theta$, for $k=1,2, \ldots, n+1$, with $\theta_{n+1}=\theta_{B}, \Delta \theta$ thus being the uniform increment over the po- 


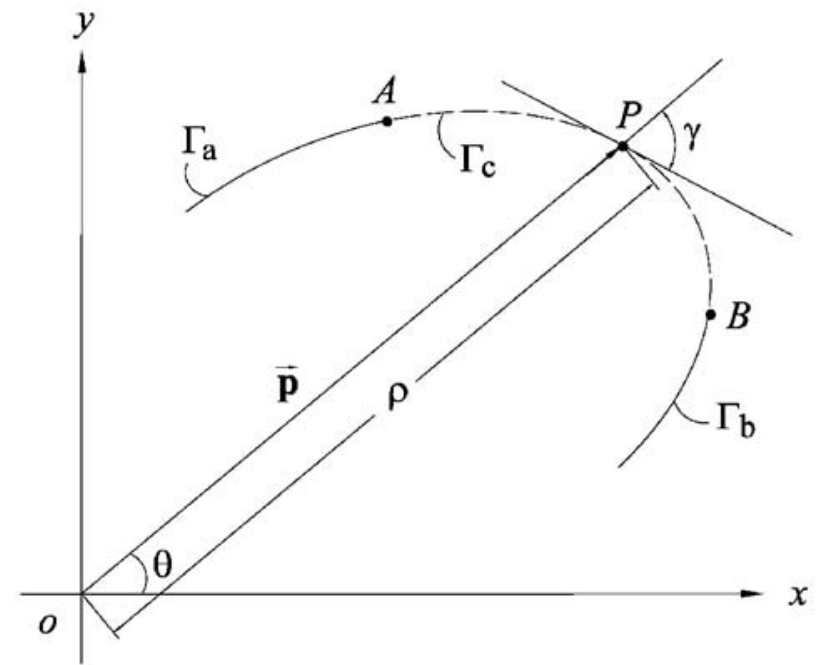

Fig. 1: Blending of two curve segments with a third one

lar coordinate $\theta$. In defining the polar coordinates of the blending curve $\Gamma_{c}$, care must be taken to choose the origin conveniently, so as to avoid more than one point with the same $\theta$-coordinate, which would render the discretization adopted here invalid. Under these conditions, then we assume that a function $\rho=\rho(\theta)$ exists in the interval $\left[\theta_{a}, \theta_{B}\right]$.

Further, we define the $n+2$-dimensional vectors, $\rho, \rho^{\prime}$ and $\rho^{\prime \prime}$, namely,

$$
\begin{aligned}
& \rho=\left[\rho_{0}, \rho_{1}, \ldots, \rho_{n}, \rho_{n+1}\right]^{T} \\
& \rho^{\prime}=\left[\rho_{0}^{\prime}, \rho_{1}^{\prime}, \ldots, \rho_{n}^{\prime}, \rho_{n+1}^{\prime}\right]^{T} \\
& \rho^{\prime \prime}=\left[\rho_{0}^{\prime \prime}, \rho_{1}^{\prime \prime}, \ldots, \rho_{n}^{\prime \prime}, \rho_{n+1}^{\prime \prime}\right]^{T}
\end{aligned}
$$

Letting $\rho_{k}(\theta)$ be the cubic polynomial between two consecutive supporting points $P_{k}\left(\rho_{k}, \theta_{k}\right)$ and $P_{k+1}\left(\rho_{k+1}, \theta_{k+1}\right)$, we have

$$
\begin{gathered}
\rho_{k}(\theta)=A_{k}\left(\theta-\theta_{k}\right)^{3}+B_{k}\left(\theta-\theta_{k}\right)^{2}+C_{k}\left(\theta-\theta_{k}\right)+D_{k}, \\
\theta_{k} \leq \theta \leq \theta_{k+1}, 0 \leq k \leq n .
\end{gathered}
$$

By virtue of the $G^{2}$-continuity of non-parametric cubic splines, a linear relationship between $\rho$ and $\rho^{\prime}$ exists, namely,

$$
\mathbf{A} \rho^{\prime \prime}=6 \mathbf{C} \boldsymbol{\rho},
$$

where both $\mathbf{A}$ and $\mathbf{C}$ are $n \times(n-1)$ matrices. Further, we recall the expressions for the angle $\gamma$ made by the tangent to the curve with the radius vector, and the curvature, in polar coordinates:

$$
\begin{aligned}
& \tan \gamma=\frac{\rho}{\rho^{\prime}}, \\
& \kappa=\frac{\rho^{2}+2\left(\rho^{\prime}\right)^{2}-\rho \rho^{\prime \prime}}{\left(\rho^{2}+\left(\rho^{\prime}\right)^{2}\right)^{\frac{3}{2}}},
\end{aligned}
$$

where

$$
\begin{aligned}
& \rho^{\prime}=\frac{\mathrm{d} \rho}{\mathrm{d} \theta}, \\
& \rho^{\prime \prime}=\frac{\mathrm{d} \rho^{\prime}}{\mathrm{d} \theta} .
\end{aligned}
$$

Moreover, let $t_{k}$ and $\kappa_{k}$ denote $\tan \gamma$ and $\kappa$ at $P_{k}$, i.e.,

$$
\begin{aligned}
t_{k} & =\frac{\rho_{k}}{\rho_{k}^{\prime}}, \\
\kappa_{k} & =\frac{\rho_{k}^{2}+2\left(\rho_{k}^{\prime}\right)^{2}-\rho_{k} \rho_{k}^{\prime \prime}}{\left(\rho_{k}^{2}+\left(\rho_{k}^{\prime}\right)^{2}\right)^{\frac{3}{2}}} .
\end{aligned}
$$

That is, $t_{k}=t_{k}\left(\rho_{k}, \rho_{k}^{\prime}\right)$ and $\kappa_{k}=\kappa_{k}\left(\rho_{k}, \rho_{k}^{\prime}, \rho_{k}^{\prime \prime}\right)$. Let us now introduce two end-conditions:

$$
t_{0}=t_{A}=\tan \gamma_{A}, t_{n+1}=t_{B}=\tan \gamma_{B},
$$

where $\gamma_{A}$ and $\gamma_{B}$ are the known tangent angles at point $A$ and $B$ of $\Gamma_{a}$ and $\Gamma_{b}$, respectively, matrices $\mathbf{A}$ and $\mathbf{C}$ then becoming square.

A linear relation between $\rho$ and $\rho^{\prime}$ also exist, namely,

$$
\mathbf{P} \boldsymbol{\rho}^{\prime}=\mathbf{Q} \boldsymbol{\rho},
$$

where $\mathbf{P}$ and $\mathbf{Q}$ are $(n+1) \times(n+2)$ matrices. Expressions for all four matrices $\mathbf{A}, \mathbf{C}, \mathbf{P}$ and $\mathbf{Q}$ are included in the Appendix.

Hence, slope and curvature values at the unknown supporting points $\left\{P_{k}\right\}_{1}^{n}$ of $\Gamma_{c}$ can all be expressed as functions of $\left\{\rho_{k}\right\}_{1}^{n}$. In formulating the curve-synthesis problem within the realm of optimum design, we let $x$ be the vector of design of variables, namely,

$$
\boldsymbol{x}=\left[\rho_{1} \ldots \rho_{n}\right]^{T} .
$$

Notice that

$$
\rho_{A}=\rho_{0}, \rho_{B}=\rho_{n+1},
$$

which are known, and part of the data. We can now formulate the curve-synthesis problem at hand as an optimum-design problem:

$$
z=\frac{1}{n} \sum_{1}^{n} w_{k} k_{k}^{2} \rightarrow \min _{x} .
$$

Where $w_{k}$ is the normal weight at the $k^{\text {th }}$ supporting point, whenever one needs to assign different importance to different points. We term the weights normal because they obey the relation

$$
\sum_{1}^{n} w_{k}=1
$$

the problem being constrained to obey the $G^{2}$-continuity conditions at the two blending points. Specifically, these constraints are,

$\kappa_{0}\left(\boldsymbol{\rho}, \boldsymbol{\rho}^{\prime}, \boldsymbol{\rho}^{\prime \prime}\right)=\kappa_{A}, \kappa_{n+1}\left(\boldsymbol{\rho}, \boldsymbol{\rho}^{\prime}, \boldsymbol{\rho}^{\prime \prime}\right)=\kappa_{B}$,

where $\kappa_{A}$ and $\kappa_{B}$ are the curvature values of curve $\Gamma_{a}$ at point $A$ and of curve $\Gamma_{b}$ at point $B$, respectively, thereby formulating an equality constrained optimum-design problem. Furthermore, special cases may call for additional constraints. For example, if convexity is to be imposed, then we must add the inequality constraints

$$
\kappa_{k} \geq 0, k=1,2, \ldots, n .
$$

Once the optimization problem is formulated, a solution is possible with scientific code available in the market. We resort to our own Orthogonal Decomposition Algorithm, implemented in the ODA package [9], which is a library of $\mathrm{C}$ routines, especially suited to solve nonlinear least-square problems. 


\section{Examples}

Several shape-synthesis problems have been solved using cubic-splines. Two examples belonging to structural optimization are outlined in the balance of this paper.

\subsection{Synthesis of the neutral axis of a curved robotic link}

The first case arose during the design of a novel spherical parallel robot, the "Agile Wrist" [10], as shown in Fig. 2. As a module of an 11-degree-of-freedom (dof) long-reach robot operating on fragile objects such as the fuselage of aircraft, the wrist requires a high positioning accuracy since any error in the positioning of the tool could lead to expensive damage.

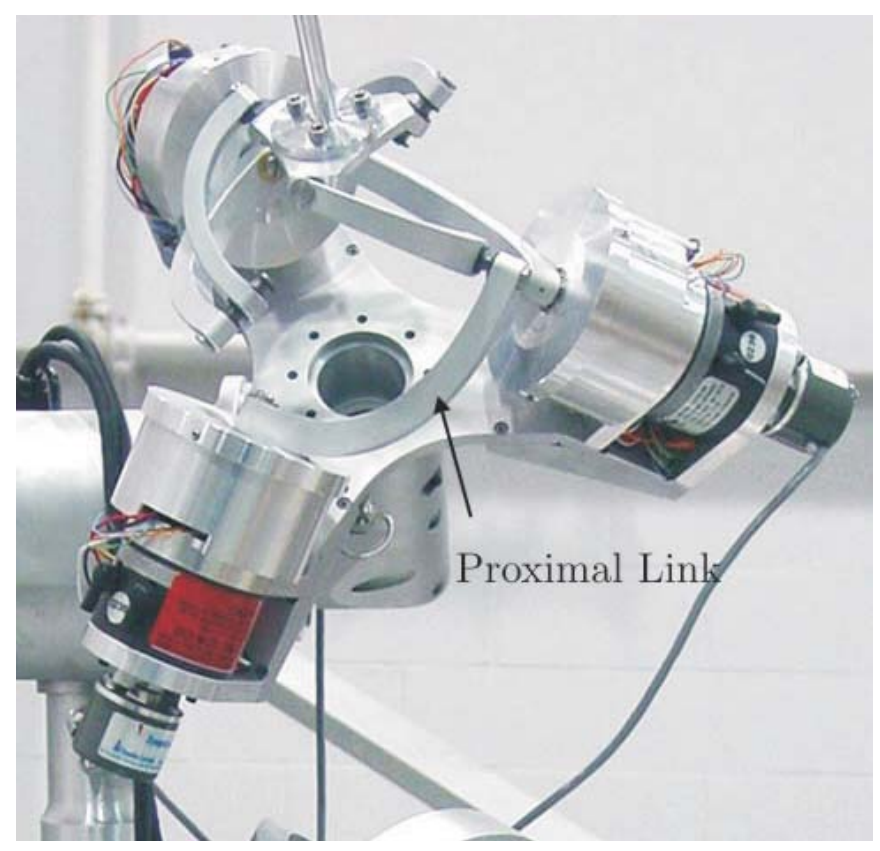

Fig. 2: Prototype of the Agile Wrist

The kinematic chain of the "Agile Wrist" was borrowed from the design of the "Agile Eye", developed at Laval University in Quebec City [11]. Many factors can affect robot accuracy, such as manufacturing and assembly errors, calibration errors, etc. Our concern is the flexibility of the manipulator links, which significantly reduces the positioning accuracy of the robot due to external and inertial loading. For the most part, the geometric shape and the cross-section dimensions of robot links of manipulators are designed arbitrarily, with simple shapes and constant cross sections. Current structural optimization technology and the use of highly accurate CNC machine tools enable designers to produce optimum, if less obvious, shapes; an efficient selection of the design parameters should improve link stiffness without making the link heavier. A general trend in the design of lighter and stiffer structures has led to the use of sophisticated materials, like carbon fibre-reinforced composites, with the consequent slendering of structural components. These components should ensure that no failure can occur under a given range of loads, which has motivated intensive research in the area of structural optimization, a very active field since Schmidt set forth an approach for coupling finite element analysis and nonlinear programming [12]. In our work, structural optimization was conducted through the optimum design of the links of the Agile Wrist in order to enhance the load-carrying capacity, while minimizing weight. We focus here on the shaping of the links, rather than on material selection. The material of choice, as decided on earlier, was aluminum AL2014.

For optimization purposes, the problem at hand was decomposed into two main steps: The first one consists in defining the neutral axis of the links, i.e., the mid-curve; the second consists in defining the cross section at each point of the mid-curve. The idea is rather simple: once the mid-curve of the link is defined, the link shape is obtained by sweeping and blending simultaneously a cross-section of a given shape (rectangle, circle, etc.), whose dimensions are variable along the curve, and lie in a plane normal to the mid-curve. Since the second step can be completed with CAE software, we focus on the synthesis of the mid-curve. To this end, the mid-curve $\Gamma$ of the proximal link, shown in Fig. 3, is defined so as to minimize the root-mean square value of the curvature throughout $\Gamma$ and to ensure a blending of the linear segments with the curved segment as smooth as possible. Notice that the two ends lie at different distances from the center of the wrist, in order to increase the workspace of the Agile Wrist, besides reducing the likelihood of collisions among moving links and the tool installed on top of the upper platform.

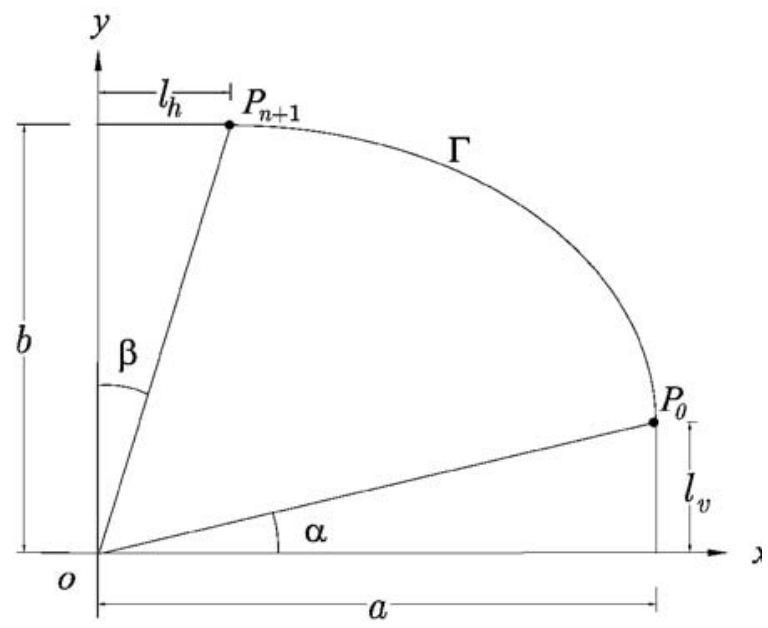

Fig. 3: The mid-curve of the proximal link

Twenty spline supporting points $P_{i}$, for $i=0, \ldots, 19$, are used, whose angles $\theta_{i}$ in polar coordinates are given by:

$$
\theta_{i}=\alpha+\frac{i}{n+1}\left[\frac{\pi}{2}-(\alpha+\beta)\right]
$$

with

$$
\alpha=\arctan \left(\frac{l_{v}}{a}\right) \text { and } \beta=\arctan \left(\frac{l_{h}}{b}\right) .
$$

With the aid of ODA, the optimum values of the design variables were found. The objective function to minimize is the rms value of the array of curvature values at the supporting points, while respecting the tangency and zero-curvature conditions at points $P_{0}$ and $P_{n+1}$. A convexity condition was added, so as to ensure that no changes in curvature-sign oc- 
Table 1: Numerical results for the optimum mid-curve of the proximal link

\begin{tabular}{|c||c|c|c||c||c|c|c|}
\hline$i$ & $\rho_{i}[\mathrm{~mm}]$ & $x_{i}[\mathrm{~mm}]$ & $y_{i}[\mathrm{~mm}]$ & $i$ & $\rho_{i}[\mathrm{~mm}]$ & $x_{i}[\mathrm{~mm}]$ & $y_{i}[\mathrm{~mm}]$ \\
\hline \hline 0 & 90.3009 & 89.5000 & 12.0000 & 10 & 87.7878 & 60.7257 & 63.3962 \\
\hline 1 & 91.2159 & 89.3859 & 18.1795 & 11 & 86.3873 & 55.4222 & 66.2658 \\
\hline 2 & 91.9843 & 88.7004 & 24.3585 & 12 & 84.9587 & 49.9954 & 68.6910 \\
\hline 3 & 92.4646 & 87.3132 & 30.4319 & 13 & 83.5441 & 44.5046 & 70.7032 \\
\hline 4 & 92.6339 & 85.2225 & 36.3065 & 14 & 82.1737 & 38.9943 & 72.3323 \\
\hline 5 & 92.4845 & 82.4522 & 41.8929 & 15 & 80.8669 & 33.4957 & 73.6036 \\
\hline 6 & 92.0253 & 79.0508 & 47.1128 & 16 & 79.6338 & 28.0313 & 74.5372 \\
\hline 7 & 91.2811 & 75.0882 & 51.9039 & 17 & 78.4782 & 22.6175 & 75.1484 \\
\hline 8 & 90.2919 & 70.6501 & 56.2245 & 18 & 77.4004 & 17.2674 & 75.4497 \\
\hline 9 & 89.1084 & 65.8309 & 60.0549 & 19 & 76.4477 & 12.0000 & 75.5000 \\
\hline
\end{tabular}

cur. These twenty points, expressed first in polar coordinates, were then expressed in Cartesian coordinates as required

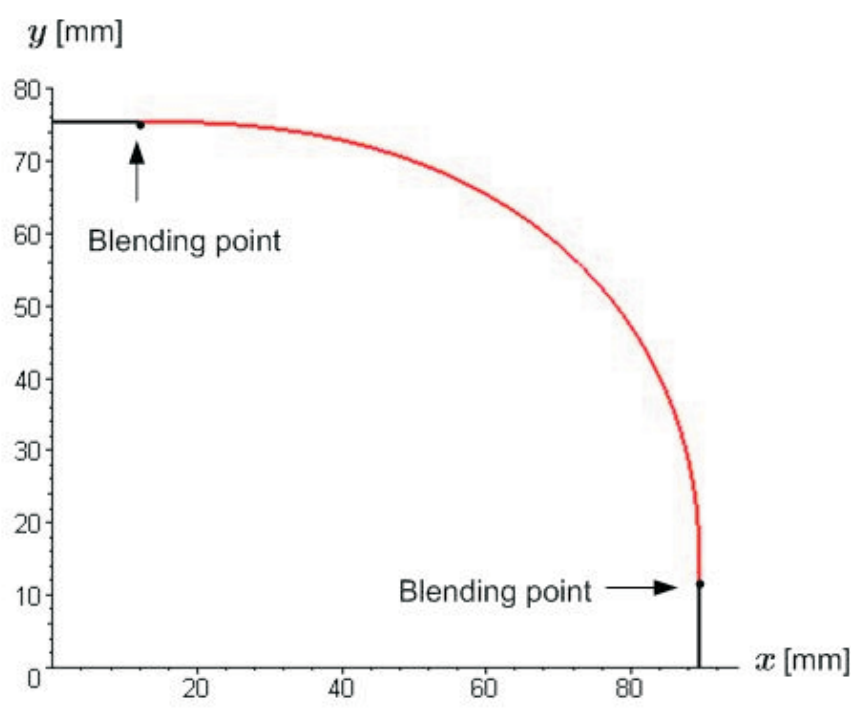

(a)

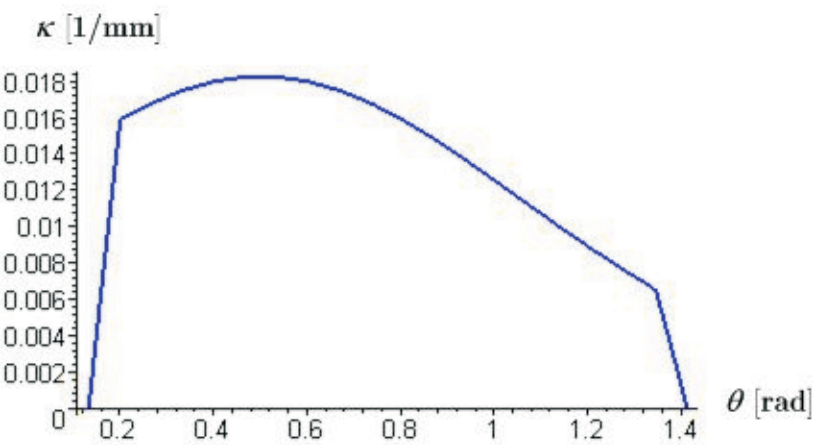

(b)

Fig. 4: Mid-curve synthesized for the Agile Wrist: (a) the curve, (b) its curvature distribution

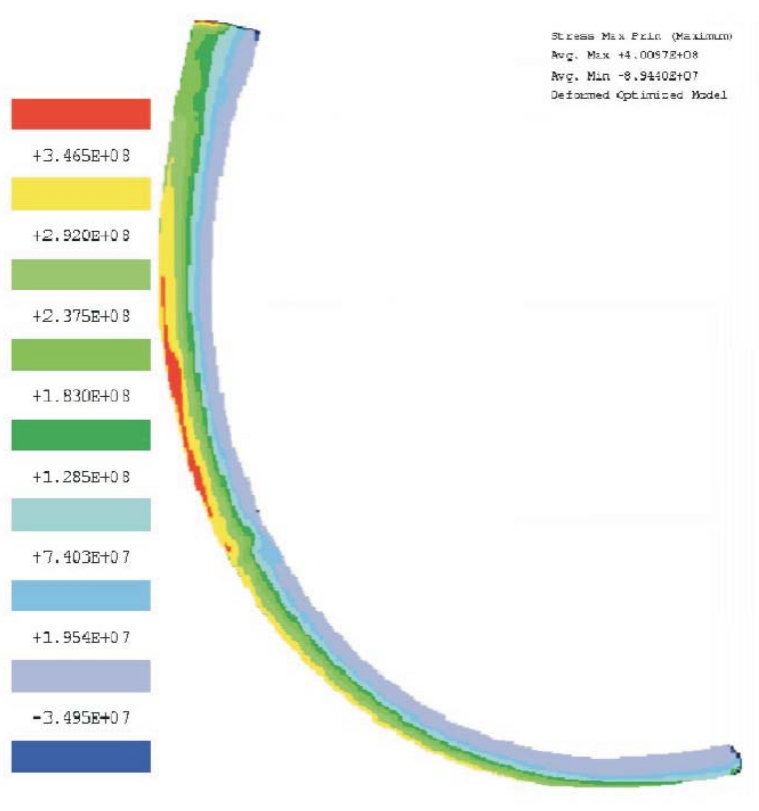

Fig. 5: Von Mises stress distribution along the optimized proximal link (thickest cross-section is located closest to the motor)

by CAE software. The radius $\rho_{i}$ and the Cartesian coordinates $\left(x_{i}, y_{i}\right)$ obtained for $a=89.5 \mathrm{~mm}, b=75.5 \mathrm{~mm}$ and $l_{v}=l_{h}=12.0 \mathrm{~mm}$, are listed in Table 1 . The rms value of the curvature distribution over the synthesized curve is $0.0144 \mathrm{~mm}^{-1}$. The curve is plotted in Fig. $4 \mathrm{a}$, its curvature distribution being shown in Fig. $4 \mathrm{~b}$ for verification of the curvature continuity at the two blending points. Based on this mid-curve, structural optimization was completed by considering the cross-section at each point of the curve. The overall stress analysis on an optimized link with two circular cross-sections at its ends is graphically displayed in Fig. 5, where no stress concentrations are observed. It is noted that the link fabricated, shown in Fig. 2, exhibits a rectangular, uniform cross-section, which is cheaper to machine, and we decided to adopt to stay within budget. 


\subsection{The design of a wrist mechanism housing}

The second example pertains to an innovative mechanism, a gearless pitch-roll wrist (PRW) [13], namely, a robotic device intended to produce two-degree-of-freedom rotations of a robotic gripper about a fixed point, the wrist center. Conventional means of producing such motions rely on a bevel-gear differential train, similar to those found in automotive driving-wheel axes. Moreover, such trains, in robotic wrists, invariably bear straight-tooth bevel gears, which are the source of noise and significant power losses. In an attempt to overcome these drawbacks, a PRW is being designed at the Robotic Mechanical Systems Laboratory, McGill University, Montreal, as depicted in Fig. 6a, with gears replaced by cam-roller pairs.

The key component of the mechanism is an array of spherical Stephenson mechanisms (SSMs) used to transmit the power from the two independent cam rotations to the gripper, as illustrated in Fig. 6b. The two cams are driven by the motion of the two roller-carrying disks of Fig. 6a, which are rigidly coupled to their respective motors.

When the two face-to-face motors turn at opposite angular velocities of identical absolute values, the whole array turns about the common axis of the two cams, as a single rigid body (pitch); when the two motors turn at identical angular velocities, the plane containing the four spherical-linkage centers remains stationary, but the gripper turns about its axis (roll). The array must be supported by a housing that doubles as a protection means to isolate the spherical linkages from the environment dust and dirt.

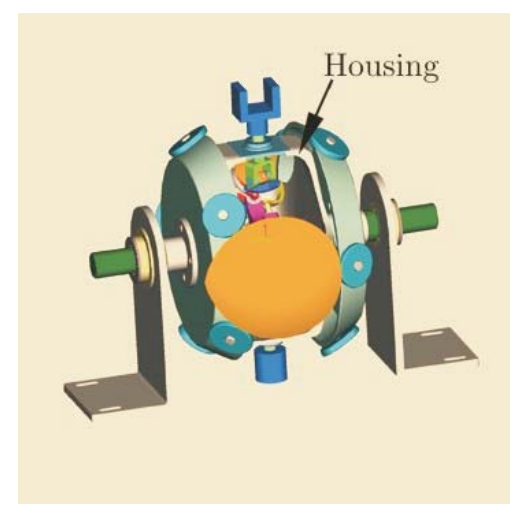

(a)

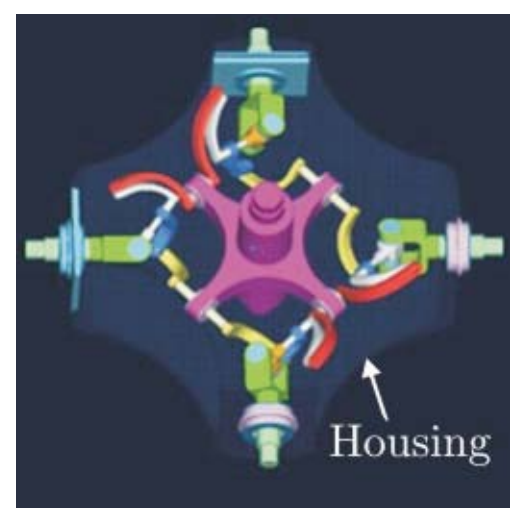

(b)

Fig. 6: An innovative PRW with cam-roller pairs: (a) the mechanism; (b) an array of two SSMs and their mirror-images
One approach in designing the housing is to make use of Lamé curves [14], which are given by the implicit function

$$
\left|\frac{x}{a}\right|^{p}+\left|\frac{y}{b}\right|^{p}=1,
$$

where $p$ is an integer and $a$ and $b$ are real numbers that determine the dimensions, $2 a \times 2 b$, of the box circumscribing the curve. A PRW housing design [15] is shown in Fig. 7, which is based on Lamé curves, with $p=4$ for the inner surface, and $p=6$ for its outer counterpart. In most common applications, Lamé curves are needed only in the first quadrant, at which we can dispense with the absolute-value signs, and rewrite eq. (21) as

$$
\left(\frac{x}{a}\right)^{p}+\left(\frac{y}{b}\right)^{p}=1,0 \leq x \leq 1,0 \leq y \leq 1 .
$$

In any event, moreover, the curvature of these curves at the points of intersection with the coordinate axes vanishes, thereby allowing for $G^{2}$-continuity at the points of blending with two line segments at $90^{\circ}$.

While Lamé curves, in the foregoing case, provide a thicker cross section at the points of maximum curvature, a plus for

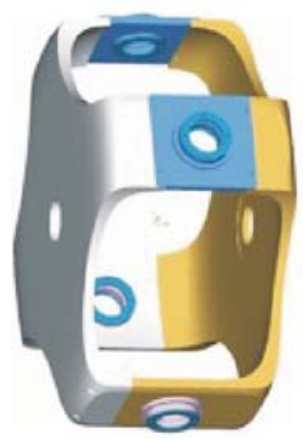

Fig. 7: The Stephenson-linkage housing made up of two identical covers

the curves, the additional material, placed at a distant location from the cam axis, adds significantly to the moment of inertia of the whole device.

An alternative design is thus desirable to replace the current housing shape by means of a new shape that is free of the drawbacks of Lamé curves. The new housing is to have both uniform thickness and zero curvature at the blending points with the straight bearing housings.

The profile of a new design is displayed in Fig. 8a, which was generated based on non-parametric cubic splines. The numerical results, namely, the values of the $\rho$-coordinate at the unknown supporting points, are listed in Table 2. In this case, $a=198 \mathrm{~mm}, b=98 \mathrm{~mm}$, the half-length of the horizontal line being equal to $100 \mathrm{~mm}$ and the half-length of its vertical counterpart being equal to $20 \mathrm{~mm}$. A Lamé curve with $p=4$ is displayed in Fig. 8a together with the synthesized curve for comparison. The curvature distributions of the two curves are shown in Fig. 8b, which indicates that the synthesized curve has a lower maximum curvature value, implying a higher allowable bending moment. The rms value of the curvature of the synthesized curve is $0.0119 \mathrm{~mm}^{-1}$, as compared with $0.0142 \mathrm{~mm}^{-1}$ of the Lamé curve with $p=4$. A new housing is currently under design. 


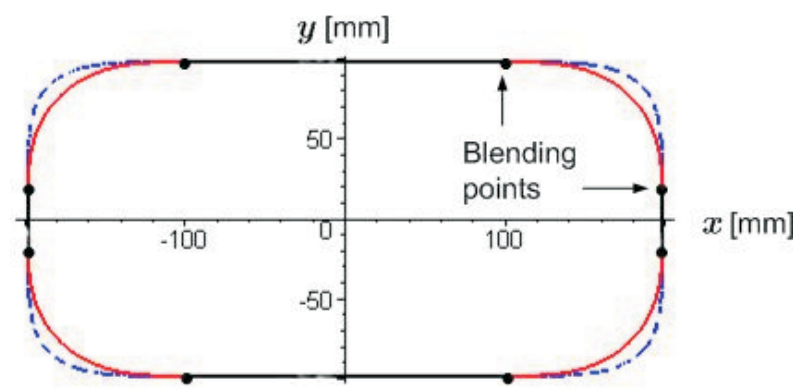

(a)

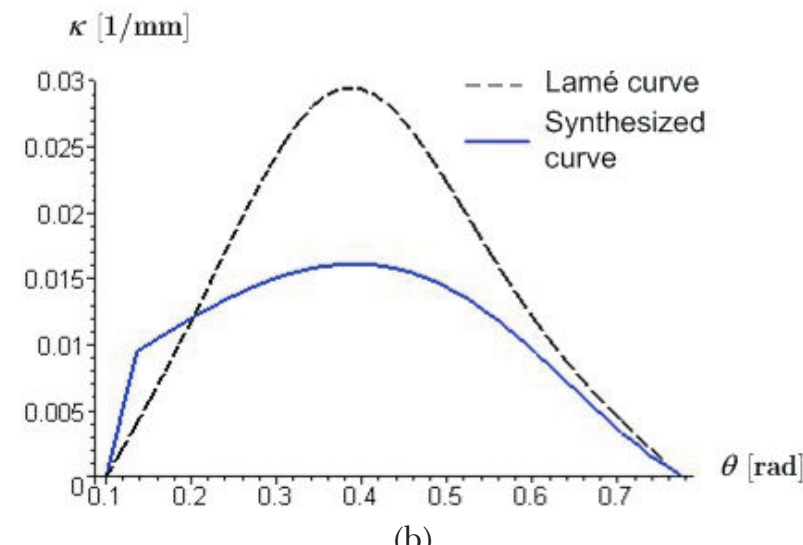

Fig. 8: (a) New housing profile and (b) curvature distribution over the synthesized curve; shown dashed is the Lamé curve

Table 2: Numerical results for the housing design

\begin{tabular}{|c|c|c||c|c|c|}
\hline$i$ & $\rho_{i}[\mathrm{~mm}]$ & $\kappa_{i}$ & $i$ & $\rho_{i}[\mathrm{~mm}]$ & $\kappa_{i}$ \\
\hline \hline 0 & 199.0075 & 0.0000 & 10 & 189.4187 & 0.0155 \\
\hline 1 & 199.7687 & 0.0094 & 11 & 185.4056 & 0.0146 \\
\hline 2 & 200.3707 & 0.0109 & 12 & 180.7433 & 0.0133 \\
\hline 3 & 200.6720 & 0.0122 & 13 & 175.4735 & 0.0117 \\
\hline 4 & 200.6039 & 0.0135 & 14 & 169.6913 & 0.0098 \\
\hline 5 & 200.1041 & 0.0145 & 15 & 163.5592 & 0.0076 \\
\hline 6 & 199.1170 & 0.0153 & 16 & 157.2998 & 0.0054 \\
\hline 7 & 197.5937 & 0.0159 & 17 & 151.1588 & 0.0033 \\
\hline 8 & 195.4919 & 0.0161 & 18 & 145.3498 & 0.0014 \\
\hline 9 & 192.7760 & 0.0160 & 19 & 140.0142 & 0.0000 \\
\hline
\end{tabular}

\section{Conclusions}

A methodology of curve synthesis with $G^{2}$-continuity was proposed, based on non-parametric cubic splines. Two synthesis examples are provided to demonstrate the effectiveness of the methodology.

The proposed methodology was applied to planar curves. It can also be used in spatial curves, if these curves are synthesized via their projections on the orthogonal planes [16]. For shape optimization, the procedure described herein can be integrated with CAE software. The procedure is also expected to find applications in areas such as trajectory generation and path planning for manipulators and mobile robots.

\section{References}

[1] Angeles, J.: Synthesis of Plane Curves with Prescribed Geometric Properties Using Periodic Splines. Computer-Aided Design, Vol. 15 (1983), No. 3, p. 147-155.

[2] Böhm, W., Farin, G., Kahmann, J.: A Survey of Curve and Surface Methods in CAGD. Computer Aided Geometric Design, Vol. 1 (1984), No. 1, p. 1-60.

[3] Hosaka, M.: Modeling of Curves and Surfaces in CAD/CAM. Springer-Verlag, 1996.

[4] Hoschek, J., Lasser, D.: Fundamentals of Computer Aided Geometric Design. Springer-Verlag, 1993.

[5] Lin, P. D., Lin, M. F.: Geometric Modelling of an Elliptic Ball-End Mill. Proc. IMechE - Part B, Vol. 219 (2005), No. 1, p. 87-97.

[6] Pottmann, H.: Industrial Geometry: Recent Advances and Applications in CAD. Computer Aided Design, Vol. 37 (2005), No. 7, p. 751-766.

[7] Neuber, H.: Theory of Notch Stresses: Principles for Exact Calculation of Strength with Reference to Structural Form and Material. U.S. Dept. of Commerce, Office of Technical Services, Washington, 1961.

[8] Pottmann, H., Wallner, J.: Computational Line Geometry. Springer-Verlag, Heidelberg, Berlin, 2001.

[9] Teng, C. P., Angeles, J.: A Sequential-Quadratic-Programming Algorithm Using Orthogonal Decomposition with Gerschgorin Stabilization. ASME J. of Mechanical Design, Vol. 123 (2001), No. 4, p. 501-509.

[10] Bidault, F., Teng, C. P., Angeles, J.: Structural Optimization of a Spherical Parallel Manipulator Using a Two-Level Approach. Proc. of ASME 2001 Design Engineering Technical Conferences, 2001, \#DETC 2001-21030.

[11] Gosselin, C.M., Hamel, J.-F.: The Agile Eye: A High-Performance Three-Degree-of-Freedom Camera Orienting Device. IEEE Int. Conf. on Robotics and Automation, 1994, p. 781-786.

[12] Schmidt, L. A.: Structural Design by Systemastic Synthesis. Proc. $2^{\text {nd }}$ ASCE Conf. Electronic Computation, Pittsburgh, Pennsylvania, USA, 1960, p. 1105-1122.

[13] Hernandez, S., Bai, S., Angeles, J.: The Design of a Chain of Stephenson Mechanisms for a Gearless Pitch-Roll Wrist. Proc. ASME Design Engineering Technical Conferences (DETC'04), Salt Lake City, Utah, USA, 2004, \#DETC 2004-57424.

[14] Gardner, M.: The Superellipse: a Curve that Lies Between the Ellipse and the Rectangle. Scientific American, Vol. 21 (1965), p. 222-234.

[15] Hernandez, S.: Optimum Design of Epicyclic Trains of Spherical Cam-Roller Pair. Master's thesis, McGill University, Montreal, Canada, 2004.

[16] Angeles, J., Akhras, R., Liu, Z.: The Synthesis of Smooth Trajectories for Pick-and-Place Operations of Spherical Wrists. Mechanism and Machine Theory, Vol. 28 (1992), No. 2, p. 261-269. 
Chin-Pun Teng

Department of Technical R\&D

The Original Cakerie Ltd, B.C.,

Canada

Shaoping Bai

e-mail:shb@ime.aau.dk

Department of Mechanical Engineering

Aalborg University, Denmark

Jorge Angeles

e-mail: angeles@cim.mcgill.ca

Department of Mechanical Engineering \& Centre for

Intelligent Machines

McGill University, Montreal, Canada

\section{Appendix}

The linear relationship between $\rho$ and $\rho^{\prime \prime}$ is expressed as

$$
\mathbf{A} \boldsymbol{\rho}^{\prime \prime}=6 \mathbf{C} \boldsymbol{\rho}
$$

where

$$
\mathbf{A}=\Delta \theta\left[\begin{array}{ccccccc}
2 & 1 & 0 & 0 & \cdots & 0 & 0 \\
1 & 4 & 1 & 0 & \cdots & 0 & 0 \\
0 & 1 & 4 & 1 & \cdots & 0 & 0 \\
\vdots & \vdots & \vdots & \vdots & \cdots & \vdots & \vdots \\
\vdots & \vdots & \vdots & \vdots & \cdots & \vdots & \vdots \\
0 & 0 & \cdots & 1 & 4 & 1 & 0 \\
0 & 0 & 0 & \cdots & 1 & 4 & 1 \\
0 & 0 & 0 & \cdots & 0 & 1 & 2
\end{array}\right], \quad \mathbf{C}=\frac{1}{\Delta \theta}\left[\begin{array}{ccccccc}
c_{11} & 1 & 0 & 0 & \cdots & 0 & 0 \\
1 & -2 & 1 & 0 & \cdots & 0 & 0 \\
0 & 1 & -2 & 1 & \cdots & 0 & 0 \\
\vdots & \vdots & \vdots & \vdots & \cdots & \vdots & \vdots \\
\vdots & \vdots & \vdots & \vdots & \cdots & \vdots & \vdots \\
0 & 0 & \cdots & 1 & -2 & 1 & 0 \\
0 & 0 & 0 & \cdots & 1 & -2 & 1 \\
0 & 0 & 0 & \cdots & 0 & 1 & c_{n n^{\prime \prime}}
\end{array}\right],
$$

in which $n^{\prime \prime}=n+2$ and

$$
c_{11}=-1-\frac{\Delta \theta}{t_{A}}, \quad c_{n n^{\prime \prime}}=-1-\frac{\Delta \theta}{t_{B}} .
$$

Similarly, the linear relationship between $\rho$ and $\rho^{\prime \prime}$ is

$$
\mathbf{P} \rho^{\prime}=\mathbf{Q} \rho
$$

where

$$
\mathbf{P}=\Delta \theta\left[\begin{array}{ccccccc}
1 / \Delta \theta & 0 & 0 & 0 & \cdots & 0 & 0 \\
1 & 4 & 1 & 0 & \cdots & 0 & 0 \\
0 & 1 & 4 & 0 & \cdots & 0 & 0 \\
\vdots & \vdots & \vdots & \vdots & \cdots & \vdots & \vdots \\
\vdots & \vdots & \vdots & \vdots & \cdots & \vdots & \vdots \\
0 & 0 & \cdots & 1 & 4 & 1 & 0 \\
0 & 0 & 0 & \cdots & 1 & 4 & 1 \\
0 & 0 & 0 & \cdots & 0 & 0 & 1 / \Delta \theta
\end{array}\right], \quad \mathbf{Q}=\left[\begin{array}{ccccccc}
1 / t_{A} & 0 & 0 & 0 & \cdots & 0 & 0 \\
-3 & 0 & 3 & 0 & \cdots & 0 & 0 \\
0 & -3 & 0 & 3 & \cdots & 0 & 0 \\
\vdots & \vdots & \vdots & \vdots & \cdots & \vdots & \vdots \\
\vdots & \vdots & \vdots & \vdots & \cdots & \vdots & \vdots \\
0 & 0 & \cdots & -3 & 0 & 3 & 0 \\
0 & 0 & 0 & \cdots & -3 & 0 & 3 \\
0 & 0 & 0 & \cdots & 0 & 0 & 1 / t_{B}
\end{array}\right]
$$

It is noteworthy that when either $\gamma_{A}$ or $\gamma_{B}$ is equal to $\pi / 2, \mathbf{Q}$ becomes singular. In our procedure, however, we need not invert $\mathbf{Q}$, for all we compute is $\boldsymbol{\rho}^{\prime}=\mathbf{P}^{-1} \mathbf{Q} \boldsymbol{\rho}$. 